\title{
Efecto de las arañas (Arachnida: Araneae) como depredadoras de insectos plaga en cultivos de alfalfa (Medicago sativa) (Fabaceae) en Argentina
}

\author{
Andrea Armendano \& Alda González \\ Centro de Estudios Parasitológicos y de Vectores (CEPAVE-CONICET), 2 № 584 (1900) La Plata, Argentina; \\ aarmendano@hotmail.com, asgonzalez@cepave.edu.ar
}

Recibido 22-XI-2010. C Corregido 20-II-2011. Aceptado 23-III-2011.

\begin{abstract}
Effect of spiders (Arachnida: Araneae) as predators of insect pest in alfalfa crops (Medicago sativa) (Fabaceae) in Argentina. Spiders are predators that reduce insect pest populations in agroecosystems. Trials were conducted to measure the selectivity against different insect preys, the daily consumption, effect of predators alone and together with a known number of preys, and the indirect effect of predators on vegetation. For this, experimental units $(1 \mathrm{x} 1 \mathrm{~m})$ were used covered with a fine plastic mesh. Misumenops pallidus, Oxyopes salticus and Araneus sp. were used as generalist predators, and aphids, weevils, locusts, chrysomelids and Lepidoptera larvae as their potential preys. Among the preys offered, the spiders preferred Lepidoptera larvae compared to the other two pests groups (weevils and aphids). The maximum consumption rate was of $93.33 \%$ for Lepidoptera larvae, $25.33 \%$ for aphids and $11.67 \%$ for weevils. The Q Index values for the three species of spiders showed a positive selectivity only for defoliating larvae. O. salticus showed the highest values of consumption rates while Rachiplusia $n u$ was the most consumed. The maximum value of consumption in 24 hours was showed by $O$. salticus on $R . n u(\mathrm{C})=2.8$. The association of several species of predatory spiders increased the total number of insects captured, and also showed that the addition of spiders caused a decrease in the number of leaves damaged by the effect of lepidopterous larvae. Rev. Biol. Trop. 59 (4): 1651-1662. Epub 2011 December 01.
\end{abstract}

Key words: Araneae, pests, agroecosystems, natural enemies.

En los agroecosistemas las arañas son depredadoras de un número considerable de insectos perjudiciales (Riechert \& Bishop 1990, Young \& Edwards 1990, Nyffeler et al. 1994a, 1994b, 1999, Polis \& Strong 1996, Lang et al. 1999, Schmitz 2003, Wise 2006). Ciertas características como la de ocupar distintos microhábitats, atacar a distintas especies plaga al mismo tiempo, resistir la falta de alimento y la desecación, les permite reducir y mantener a las poblaciones de insectos plaga por debajo de los umbrales de daño económico (Pedigo 2001). Hairston et al. (1960) consideran que las poblaciones de herbívoros no están limitadas por la competencia por el alimento sino por la depredación. Sin embargo, las prácticas modernas, que implican repetidas perturbaciones químicas y físicas del ambiente, a veces no proveen las condiciones apropiadas para el establecimiento de los depredadores (Young \& Edwards 1990, Feber et al. 1998). Los herbívoros escapan entonces del control de los depredadores y se vuelven muy abundantes hasta el punto de causar daños severos en la vegetación. Si un depredador pudiera establecerse y alimentarse de estos herbívoros, su número se reduciría considerablemente. Sunderland (1999) considera que las arañas se encuentran dentro de este tipo de depredadores.

Las arañas atacan a sus presas con relación a la tasa de encuentro, y cuanto mayor es la densidad de una población presa (como ocurre en numerosos monocultivos), mayor es la proporción de ataques hacia dicha presa (Riechert 
\& Gillespie 1983). Existen numerosas investigaciones que han demostrado que las arañas pueden ser importantes agentes de control biológico de especies plaga tales como áfidos, larvas de lepidópteros, ortópteros, homópteros, algunos coleópteros y pequeños dípteros en distintos agroecosistemas (Riechert \& Lockley 1984, Nyffeler et al. 1994b, Nyffeler \& Sunderland 2003, Marc et al. 1999, Vichitbandha \& Wise 2002, Lang 2003).

La alfalfa (Medicago sativa L.) se ve comúnmente afectada por tres grupos de plagas primarias: las orugas defoliadoras y cortadoras, los gorgojos y los pulgones (Harcourt et al. 1986). Dado que los alfalfares tienen un tiempo de vida útil de cuatro a seis años, constituyen un microclima especial para numerosos insectos, entre ellos los pulgones. En Argentina son cuatro las especies de pulgones que atacan la alfalfa: Acyrthosiphon pisum (Harris), Acyrthosiphon kondoi Shinji, Therioaphis maculata (Buckton) y Aphis craccivora Koch (Harcourt et al. 1986, Belmonte et al. 2006). En el segundo grupo de plagas se encuentran las larvas de lepidópteros tales como: Colias lesbia (Fabricius), Anticarsia gemmatalis Hübner, Rachiplusia nu Guenée, Agrotis malefida Guenée, etc. (Belmonte et al. 2006). Los gorgojos (Coleoptera: Curculionidae) constituyen el tercer grupo, que se conoce como "el complejo de gorgojos de la alfalfa" que reúne alrededor de 20 especies de rostro corto (Harcourt et al. 1986, Lanteri 1994).

Existen registros de especies de insectos plaga de la alfalfa que son depredados por arañas, tal es el caso de algunos gorgojos, como Aramigus tessellatus Say (Harcourt et al. 1986, Lanteri 1994, Lanteri et al. 1998) y de ciertos áfidos (Edwards et al. 1979, Losey 1996, Losey \& Denno 1997, Toft 1999, Harwood et al. 2004). Dentro de estos últimos se destacan las especies A. kondoi y A. pisum (pulgones azul y verde de la alfalfa, respectivamente), responsables en los últimos años de graves pérdidas económicas en Argentina. Pese a esto los aportes sobre el rol depredador de la araneofauna en agroecosistemas en Argentina son escasos y referidos exclusivamente a la abundancia y diversidad de la taxocenosis de arañas, colonización y tasas de depredación en cultivos de soya (Minervino 1996, Liljesthröm et al. 2002, Cheli et al. 2006, Beltramo et al. 2006). El presente trabajo constituye una primera contribución al conocimiento del rol de las arañas dominantes en el cultivo de alfalfa (Armendano \& González 2010) como depredadoras de insectos plaga, a través de la realización de ensayos de selectividad, la estimación de tasas de consumo y del efecto de las arañas sobre el número de insectos plaga y sobre el daño ocasionado al cultivo.

\section{MATERIALES Y MÉTODOS}

Cuatro tipos de experiencias se realizaron con las especies de arañas dominantes del cultivo de alfalfa Misumenops pallidus (Keyserling, 1880), Oxyopes salticus Hentz, 1845, Araneus sp. y Lycosa poliostoma (Koch, 1847) (Armendano \& González 2010) sobre áfidos, gorgojos, larvas de lepidópteros, crisomélidos y ortópteros. Los ensayos se realizaron en jaulas experimentales estándar para realizar investigaciones con arañas en ecosistemas terrestres (Riechert \& Lawrence 1997, Beckerman et al. 1997, Schmitz 2003). Éstas consistieron en estructuras de metal de $1 \times 1 \mathrm{~m}$ con los cuatro lados verticales cubiertos con una fina malla plástica. El área basal de las jaulas fue de $1 \mathrm{~m}^{2}$ que se considera apropiada para insectos con ámbitos de tamaño de $2.5 \mathrm{~cm}$ de largo o menores (Schmitz 2004). La parte inferior de las clausuras se enterró en el suelo encerrando a la vegetación propia del cultivo, previa remoción manual de toda la fauna existente. La malla metálica que cubrió la parte superior de las jaulas permaneció abierta sólo para permitir la colocación de los ejemplares de arañas e insectos plaga utilizados en las distintas experiencias y luego permanecieron cerradas mediante grapas metálicas. Las jaulas experimentales se dispusieron al azar, en campos cultivados con alfalfa pertenecientes a la Estación Experimental de Los Hornos "Julio Hirschorn", Partido de La Plata, Argentina (345' S - 57 $7^{\circ} 57^{\prime}$ W). La distancia mínima entre los ensayos fue de 
$1.5 \mathrm{~m}$ a fin de impedir algún tipo de disturbio en la actividad de las presas debido a la proximidad de los tratamientos (Schmitz et al. 1997, Schmitz \& Suttle 2001).

\section{Recolección, cría y mantenimiento de los ejemplares utilizados en las experiencias}

Arañas: Debido a que los tres grupos plaga considerados se ubican sobre el estrato herbáceo, se eligieron las especies de arañas más abundantes sobre la vegetación del cultivo y pertenecientes a diferentes gremios, en cuanto al tipo y modo de capturar a sus presas. Los ejemplares hembras adultos fueron los que se utilizaron ya que los machos en general suelen alimentarse poco o dejan de hacerlo cuando alcanzan la madurez sexual. Los ejemplares de M. pallidus, O. salticus, Araneus sp. y $L$. poliostoma se recolectaron en campos de la Estación Experimental de Gorina, Partido de La Plata, Argentina ( $\left.34^{\circ} 53^{\prime} \mathrm{S}-58^{\circ} 05^{\prime} \mathrm{W}\right)$, y luego se trasladaron y mantuvieron bajo condiciones normales de laboratorio $\left(23^{\circ}+/-2^{\circ} \mathrm{C}\right)$. Solamente se recolectaron hembras subadultas para tener la seguridad que las mismas no estuvieran fecundadas y evitar variaciones en el consumo de presas. Además, se dispusieron aisladamente en frascos plásticos de 250mL tapados con voile, donde se colocó un algodón embebido en agua para evitar la desecación. Una vez por semana, se alimentaron con Drosophila melanogaster Meigen y Musca domestica Linneo hasta una semana previa al inicio de las experiencias, luego fueron mantenidas en ayunas.

Áfidos: Ejemplares de A. pisum (Hemiptera: Aphididae) fueron obtenidos de colonias criadas en la Cátedra de Cereales de la Facultad de Cs. Agrarias de la Universidad Nacional de La Plata. Los ejemplares libres de parasitoides y patógenos se ubicaron sobre plantas de alfalfa de unos $6 \mathrm{~cm}$ de altura, sembradas en macetas de $10 \times 15 \mathrm{~cm}$ (diámetro $\mathrm{x}$ altura). La recolonización de los pulgones a nuevas plantas de alfalfa se realizó cada dos semanas. Las colonias fueron mantenidas entre $20^{\circ}$ y $25^{\circ} \mathrm{C}$,
60 y $70 \%$ de humedad relativa y en lugares con exposición solar.

Curculiónidos: Los gorgojos (Coleoptera: Curculionidae) fueron recolectados en campos de vegetación espontánea mediante red de arrastre y se ubicaron en frascos de vidrio de $10 \times 15 \mathrm{~cm}$ (diámetro $\mathrm{x}$ altura) cubiertos con una gasa de malla fina. Se colocaron juntos unos 30 ejemplares machos y hembras, con semillas de cebada y se mantuvieron bajo condiciones normales de laboratorio. La renovación de las semillas se realizó cada 20 días. Para las experiencias se utilizaron ejemplares adultos $(5-7 \mathrm{~mm})$.

Lepidópteros: Larvas de Spodoptera frugiperda (Smith), A. gemmatalis y $R$. nu (Lepidoptera, Noctuidae) fueron obtenidas a partir de crías masivas realizadas en laboratorio y mantenidas mediante dietas artificiales (según Parra 1986). Para las experiencias se utilizaron larvas de segundo y tercer estadio (7-10 mm).

Crisomélidos y Ortópteros: Los ejemplares (Coleoptera: Chrysomelidae y Orthoptera) fueron recolectados en campos de vegetación espontánea mediante red de arrastre. Para las experiencias se utilizaron sólo ejemplares adultos.

\section{Ensayos}

Selectividad: En cada jaula experimental se ubicaron las plagas: 6 curculiónidos adultos, 30 áfidos adultos y 9 larvas de lepidópteros, según las densidades encontradas en infestaciones de plaga leves (Trumper 2001) frente a un solo ejemplar hembra adulta de M.pallidus, Araneus sp. y O. salticus por separado. El ensayo duró una semana, y día por medio se inspeccionaron los tratamientos con el fin de registrar el número y tipo de presa depredada y reponer el número inicial de presas; se realizaron 10 réplicas y cinco controles donde todas las especies de arañas fueron removidas. Además, se calculó el Índice Q=r(1-p)/p(1-r) (Jacobs 1974) para evaluar la preferencia hacia los 
distintos grupos plaga. Donde: r=es la fracción de un tipo de comida en la dieta del depredador y $\mathrm{p}=\mathrm{es}$ la fracción de esta comida en el medio. Cuando Q varía entre 0 y 1 , la selectividad del depredador hacia ese tipo de presa es negativa. Si Q es superior a 1 la selectividad es positiva.

Tasas de consumo: En cada jaula se ubicaron una de las tres especies de presas $(A$. gemmatalis, $R$. пи у $S$. frugiperda) con una densidad de 10 larvas $/ \mathrm{m}^{2}$ con un ejemplar de araña de cada especie (M. pallidus, O. salticus y Araneus sp.) por separado. Estas últimas fueron sometidas en el laboratorio a una semana de ayuno previo. Los ensayos con 10 réplicas cada uno se realizaron simultáneamente y duraron $24 \mathrm{~h}$. Transcurridas las $24 \mathrm{~h}$ se registraron los ejemplares encontrados en cada jaula.

Para estimar la tasa de consumo se siguió a Minervino (1996) $(\mathrm{C})=\mathrm{Nd}-\mathrm{Nm} / \mathrm{t}$, donde: No: es el número inicial de presas; $\mathrm{Nt}$ : es el número final de presas al cabo del tiempo t; $\mathrm{Nd}=\mathrm{No}-$ $\mathrm{Nt}$ es el número de presas desaparecidas en las jaulas experimentales; Nm: No-Nt es el número de presas muertas en las jaulas controles por causas distintas a la depredación; t: tiempo en días.

Efecto de los depredadores de forma aislada y en conjunto sobre el número de presas: Los tratamientos consistieron en colocar un conjunto de (i) presas frente a cada uno de los depredadores en forma individual (M. pallidus, O. salticus, Araneus sp. y $L$. poliostoma), (ii) las presas frente al conjunto de depredadores y (iii) los controles donde todas las especies de arañas fueron removidas. En cada jaula se colocaron las presas: lepidópteros $(\mathrm{n}=10)$, áfidos $(\mathrm{n}=10)$, curculiónidos $(\mathrm{n}=10)$, crisomélidos $(\mathrm{n}=10)$ y ortópteros $(\mathrm{n}=10)$. Las densidades de arañas fueron en todos los casos de cuatro ejemplares hembra. En los ensayos de depredadores aislados los cuatro ejemplares fueron de la misma especie, mientras que, en los ensayos de especies asociadas se colocó una araña de cada una de las especies consideradas; se realizaron cinco repeticiones y cinco controles y se trabajó con un total de 30 jaulas. El experimento se llevó a cabo durante $48 \mathrm{~h}$. Los datos registrados fueron analizados estadísticamente mediante el análisis de la varianza (ANOVA) con el programa Statgraphics plus 4.0.

Efecto indirecto de los depredadores sobre la vegetación: El experimento se llevó a cabo durante $72 \mathrm{~h}$, con 10 réplicas para cada uno de los tres tratamientos: (i) solamente plantas de alfalfa (ii) plantas y larvas defoliadoras, (iii) plantas, larvas y arañas (10 larvas de $R . n u$ en estadío 2 y 3 por planta, y un ejemplar de $O$. salticus). El daño en el cultivo se estimó teniendo en cuenta: el número promedio de hojas de cultivo dañadas. Las diferencias entre los controles y tratamientos se analizaron mediante ANOVA con el programa Statgraphics plus 4.0.

\section{RESULTADOS}

Ensayo de selectividad: Los resultados demostraron que las especies de arañas $M$. pallidus, O. salticus y Araneus sp. prefirieron las larvas de lepidópteros frente a los otros dos grupos de plaga ofrecidos (curculiónidos y áfidos) (Fig. 1). El porcentaje de consumo máximo de larvas de lepidópteros fue de $93.33 \%$, el de áfidos $25.33 \%$ y el de curculiónidos $11.67 \%$. En todos los casos correspondió a $O$. salticus. Los valores del Índice Q para las tres especies de arañas demostraron una selectividad positiva sólo para las larvas defoliadoras, mientras que para áfidos y curculiónidos presentaron valores entre 0 y 1 (selectividad negativa) (Test de student, $\mathrm{p}<0.05, \mathrm{gl}=46$ ) (Cuadro 1).

Tasas de consumo: $O$. salticus presentó los mayores valores de tasas de consumo (Cuadro 2). El segundo lugar de consumo le correspondió a la especie $M$. pallidus y finalmente a Araneus sp. $R$. nu fue la más consumida y $S$. frugiperda la que se capturó menos. El valor máximo de consumo registrado en 24h fue para $O$. salticus frente a $R$. $n u(C)=2.8$ (Test de t $\mathrm{p}<0.05, \mathrm{gl}=38$ ). El valor de consumo mínimo correspondió a Araneus sp. frente a A. $\operatorname{gemmatalis}(\mathrm{C})=1$. 

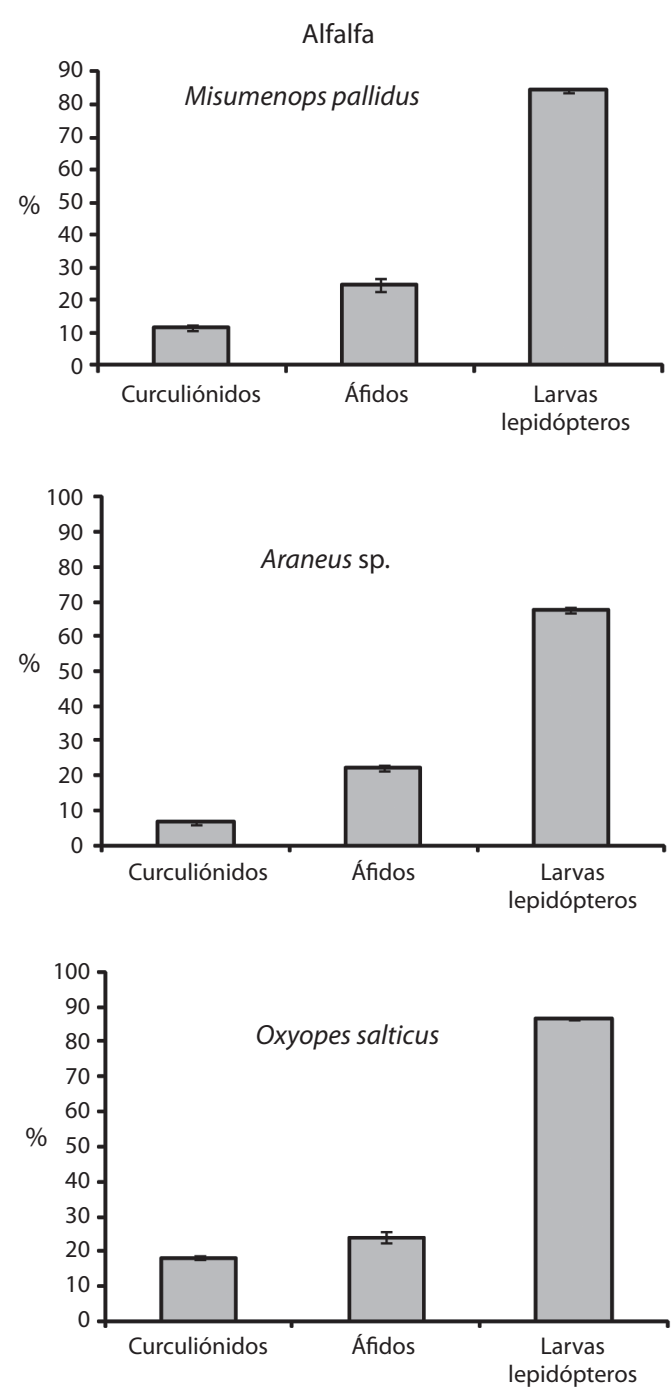

Fig. 1. Porcentaje de depredación promedio de M.pallidus, Araneus sp. y $O$. salticus en relación a cada grupo de plagas primarias en cultivos de alfalfa, en Buenos Aires, Argentina.

Fig. 1. Average predation rate of M. pallidus, Araneus sp. and $O$. salticus in relation to each group of primary pests in crops of alfalfa in Buenos Aires, Argentina.

Efecto de los depredadores de forma aislada y en conjunto sobre el número de presas: El número de insectos fue mayor en los controles, que en los tratamientos, registrándose el menor valor en los tratamientos donde se incorporaron depredadores pertenecientes a las

\section{CUADRO 1}

Valores del índice Q para los distintos grupos de plagas consumidos por M. pallidus, Araneus sp. y O. salticus

TABLE 1

Q Index values for different groups of pests consumed by M. pallidus, Araneus sp. and O. salticus

\begin{tabular}{lccc} 
& M.pallidus & Araneus $\mathrm{sp}$. & O. salticus \\
Gorgojos & $0.16 \mathrm{a}$ & $0.21 \mathrm{a}$ & $0.19 \mathrm{a}$ \\
Pulgones & $0.54 \mathrm{a}$ & $0.42 \mathrm{a}$ & $0.47 \mathrm{a}$ \\
Larvas & $3.05 \mathrm{~b}$ & $2.98 \mathrm{~b}$ & $3.22 \mathrm{c}$ \\
lepidópteros & & & \\
\hline
\end{tabular}

Las mismas letras indican que no hubo diferencias significativas (Test de $\mathrm{t}, \mathrm{p}>0.05$ ).

The same letters indicate no significant differences ( $\mathrm{t}$ test, $\mathrm{p}>0.05)$.

cuatro especies consideradas (Fig. 2). Cuando fueron colocadas en forma individual $L$. poliostoma y $O$. salticus presentaron el mayor consumo de presas. Las larvas de lepidópteros fueron las presas más consumidas. L. poliostoma fue el único depredador que consumió crisomélidos y el que consumió el mayor número de ortópteros y curculiónidos. Araneus sp. resultó el menos eficaz de los depredadores considerados. Todos los tratamientos realizados presentaron diferencias significativas con excepción de tratamientos con $O$. salticus y L. poliostoma, corroborando un efecto potenciador del conjunto de depredadores sobre las presas $(\mathrm{F}=44, \mathrm{p}<0.001)$.

Efecto indirecto de los depredadores sobre la vegetación: La adición de arañas $O$. salticus evidenció una disminución en el promedio de hojas consumidas por las larvas de $R$. $n u$ (Fig. 3). Los tres tratamientos presentaron diferencias significativas $(\mathrm{F}=130, \mathrm{p}<0.001)$.

\section{DISCUSIÓN}

Los resultados de los ensayos de selectividad muestran que las tres especies de arañas estudiadas M. pallidus, O. salticus y Araneus sp. presentaron preferencia positiva frente a las larvas de lepidópteros defoliadores, con 
CUADRO 2

Tasas de consumo diarias para las tres especies de larvas defoliadoras consumidas por M.pallidus, Araneus sp. y O. salticus

TABLE 2

Daily consumption rates for the three species of defoliating larvae consumed by $M$. pallidus, Araneus sp. and $O$. salticus

\begin{tabular}{lcccccc} 
& M.pallidus & $\pm \mathrm{SD}$ & Araneus sp. & $\pm \mathrm{SD}$ & O. salticus & $\pm \mathrm{SD}$ \\
Spodoptera frugiperda & $1.5 \mathrm{a}$ & \pm 0.53 & $1.2 \mathrm{a}$ & \pm 0.52 & $1.7 \mathrm{a}$ & \pm 0.88 \\
Anticarsia gemmatalis & $1.6 \mathrm{a}$ & \pm 0.63 & $1 \mathrm{a}$ & \pm 0.82 & $1.9 \mathrm{a}$ & \pm 0.63 \\
Rachiplusia $\mathrm{nu}$ & $2.1 \mathrm{a}$ & \pm 0.48 & $1.8 \mathrm{a}$ & \pm 0.88 & $2.8 \mathrm{~b}$ & \pm 0.79 \\
\hline
\end{tabular}

Las mismas letras indican que no hubo diferencias significativas (Test de $\mathrm{t}, \mathrm{p}>0.05$ ).

The same letters indicate no significant differences ( $t$ test, $p>0.05$ ).

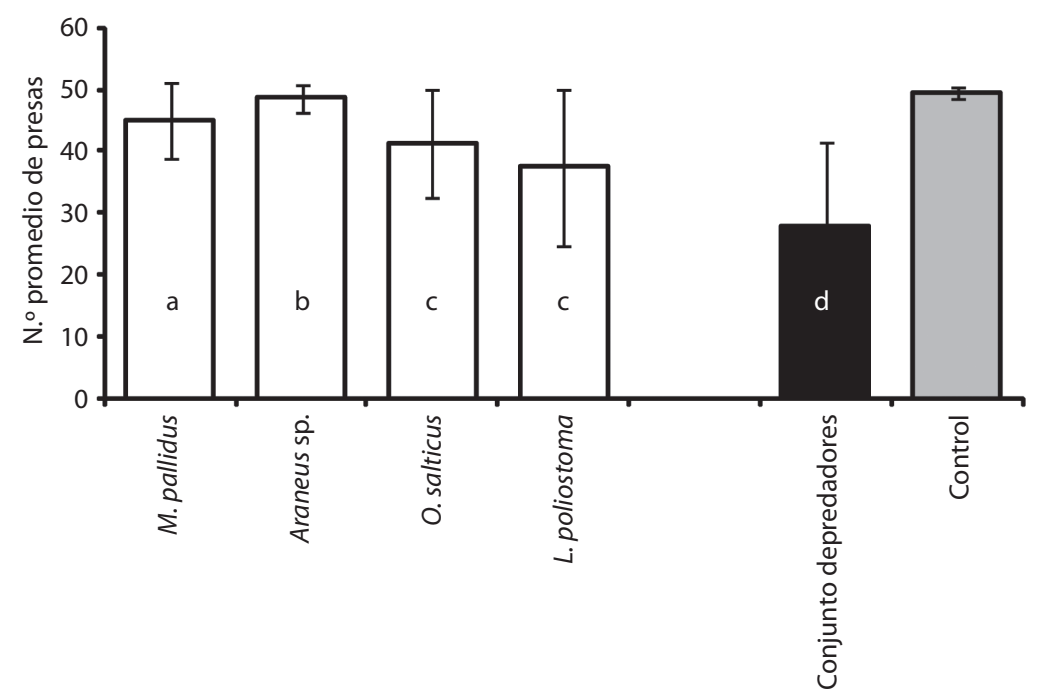

Fig. 2. Número promedio de insectos encontrados en cada tratamiento (todas las especies de arañas juntas, las especies M. pallidus, Araneus sp., O. salticus y H. poliostoma aisladas y los controles). Las mismas letras indican que no hubieron diferencias significativas $(\mathrm{p}>0.05)$.

Fig. 2. Average number of insects found on each treatment (all species of spiders together, the species M.pallidus, Araneus sp., O. salticus H. poliostoma and isolated controls). The same letters mean no significant differences ( $\mathrm{p}>0.05)$.

un escaso consumo de los otros dos grupos de plagas primarias (Aphididae y Curculionidae). Según Nyffeler \& Sunderland (2003) la dieta de las arañas en agroecosistemas incluye Diptera, Homoptera, Lepidoptera, Heteroptera, Hymenoptera y Coleoptera, que comprenden el 95\% del total de presas consumidas. También se alimentan de otras arañas, especialmente las cazadoras. Coincidentemente con los resultados obtenidos, estudios realizados mediante técnicas de radiomarcado demostraron que las arañas se alimentan preferentemente de lepidópteros plaga (huevos o larvas) en cultivos de algodón (Gravena \& Sterling 1983, Nuessly \& Sterling 1994). Las arañas involucradas son principalmente cazadoras de las familias 


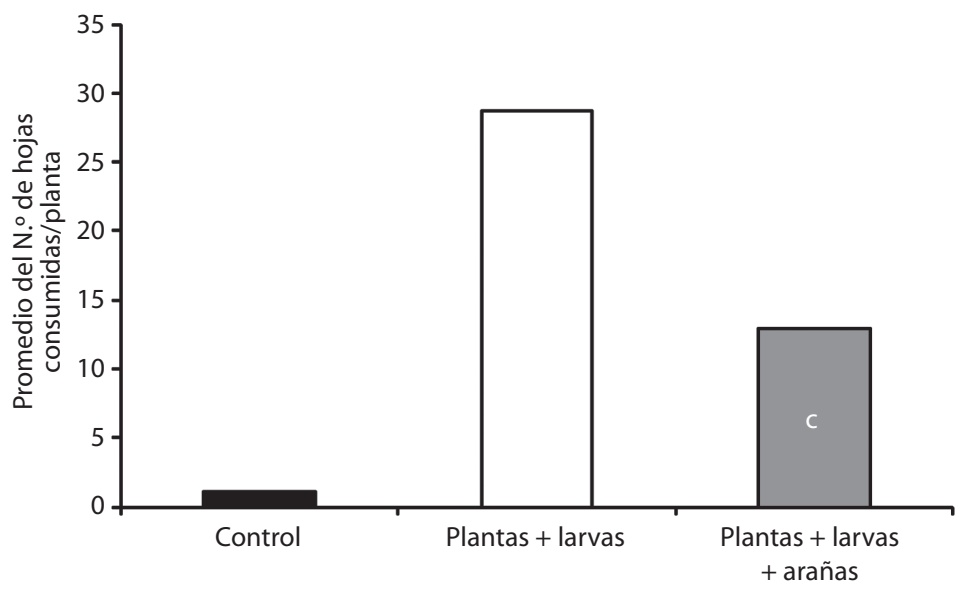

Fig. 3. Número promedio de hojas de alfalfa dañadas en $72 \mathrm{~h}$ por larvas de $R$. $n u$ por tratamiento (plantas + larvas; plantas + larvas + arañas; controles). Las letras diferentes indican que hubieron diferencias significativas $(\mathrm{p}<0.05)$.

Fig. 3. Average number of damaged alfalfa leaves in $72 \mathrm{~h}$ by larvae of $R$. $n u$ per treatment (plants + larvae; plants + larvae + spiders; controls). Different letters mean significant differences $(\mathrm{p}<0.05)$.

Oxyopidae, Salticidae, Thomisidae y Clubionidae. Estos resultados fueron verificados por estudios realizados con el método ELISA, en ensayos experimentales y a través de observaciones visuales (Ruberson \& Greenstone 1998).

En cultivos de soya se encontró también que las arañas se alimentan de varios estadios de A. gemmatalis (Godfrey et al. 1989, Gregory et al. 1989). Representantes de las familias Oxyopidae, Salticidae, Thomisidae y Clubionidae serían los principales depredadores de lepidópteros plaga en este cultivo (Reed et al. 1984, Gregory et al. 1989). Además, Shaw et al. (1987) demostraron que la remoción de depredadores que incluyen Araneidae incrementó significativamente la densidad de otros Noctuidae como Hemileuca oliviae Cockrell. En estudios realizados en algodoneros de Texas, el porcentaje de áfidos en la dieta de $O$. salticus fue baja comparado con el porcentaje de áfidos en el conjunto de presas potenciales (Nyffeler et al. 1987). Esto sugiere que los áfidos serían evitados por las arañas como presas potenciales, posiblemente porque constituyen presas de "baja calidad" para Oxyopidae y Lycosidae (Toft 1995). Esto concuerda también con los únicos ensayos realizados en nuestro país en cultivos de soya (Minervino 1996) donde también quedó demostrada una preferencia positiva de $M$. pallidus frente a larvas defoliadoras (Lepidoptera: Noctuidae) y con los ensayos llevados a cabo por Cheli et al. (2006), que demostraron un nivel de depredación intermedia (30-55\%) frente a $R$. nu y depredación negativa frente a $A$. pisum (Aphididae) y Naupactus sp. (Curculionidae) en ensayos de laboratorio.

Las arañas generalmente presentan tasas metabólicas bajas, y poseen la capacidad de reducirla en períodos de inanición (Greestone \& Bennett 1980). Turnbull (1973) determinó que la alimentación de las arañas es errática, con períodos cortos de intensa alimentación. Un sistema digestivo extenso, un abdomen distensible y la capacidad de almacenar grasas, les permite alimentarse en exceso cuando la comida es abundante, y sobrevivir sin comida por largos períodos cuando la densidad de presas disminuye (Riechert \& Harp 1987, Foelix 1982). Según Nyffeler et al. (1994b) las arañas de agroecosistemas no se alimentan continuamente, y solamente un $3.8 \%$ se han observado alimentándose en un determinado momento en 
cultivos de Europa y Estados Unidos (Nyffeler \& Sunderland 2003). Las tasas de depredación diarias dependen de distintos factores como: el tamaño de la araña, edad, sexo, eventos fisiológicos en el ciclo de vida de la araña (ej., muda) condiciones climáticas y disponibilidad y tipo de presas (Nyffeler et al. 1994a). Estos mismos autores estimaron que una araña típica de agroecosistemas captura aproximadamente una presa por día (Nyffeler et al. 1994b). En laboratorio, las mismas especies se alimentan a tasas varias veces mayores cuando el alimento es ofrecido ad libitum (Young \& Lockley 1986). Esto sugiere que las arañas en el campo consumen presas a tasas más bajas que su máxima capacidad de consumo (Nyffeler \& Sunderland 2003). En los resultados de este estudio las tasas de consumo superaron los valores estimados de una presa diaria. Probablemente, esto se relaciona con el hecho de que los ensayos si bien fueron llevados a cabo en el campo, se realizaron en jaulas experimentales donde las arañas contaron con una disponibilidad de presas constante y en densidades altas.

Algunos autores como Nentwig (1988), Nyffeler et al. (1994b) y Cheli et al. (2006), consideran que las arañas tienen una mayor predilección por las presas de dimensiones menores, de exoesqueletos más delgados, sin sustancias repelentes para los depredadores y de mayor movilidad. Esta última característica queda evidenciada en este estudio porque las tres especies de arañas estudiadas, M. pallidus, O. salticus y Araneus sp. presentaron los valores más altos de tasas de consumo frente a $R$. nu. Esta larva defoliadora, también conocida como oruga medidora, presenta un desplazamiento particular, que le da una mayor movilidad, respecto a las otras dos especies (S. Frugiperda y A. gemmatalis), quedando de alguna manera más expuesta frente a estos depredadores y especialmente frente a las arañas cazadoras, que deambulan activamente por toda la planta en busca de larvas y adultos de Lepidoptera y Heteroptera (Whitcomb 1974, Young 1989). Con respecto al tamaño de las presas mayormente consumidas, experiencias de depredación en laboratorio demuestran que el tamaño óptimo oscila entre el 50-80\% del tamaño de la araña (Nentwig 1987, Cheli et al. 2006), también evidenciado en observaciones a campo (Nyffeler et al. 1994a). Coincidentemente con LeSar \& Unzicker (1978) y Young \& Edwards (1990) en los cultivos estudiados predominaron también las arañas pequeñas, contando por lo tanto con una gran disponibilidad de presas potenciales.

Las arañas estudiadas presentaron preferencia negativa frente a los otros dos grupos plaga considerados (Aphididae y Curculionidae), relacionándose el primer caso con la presencia de glándulas pestilentes, que resultan una buena defensa frente a los depredadores (Richards \& Davies 1984, Nentwig 1987) y la segunda a la presencia de exoesqueletos fuertemente esclerotizados que dificulta la inoculación del veneno. El consumo de insectos con cutículas muy gruesas como los coleópteros, es realizado por las arañas de gran tamaño, que en general aparecen en bajo número en los agroecosistemas. Las arañas pequeñas (ej., $O$. salticus) que predominan en estos sistemas tienen quelíceros que no pueden atravesar cutículas muy gruesas y por lo tanto su contribución al efecto de depredación puede considerarse bastante escaso (Nyffeler et al. 1994a).

Riechert \& Lockley (1984) propusieron que los depredadores generalistas podrían ejercer un mayor control sobre las poblaciones de presas, si éstos se presentan en asociaciones de varias especies en vez de especies aisladas. Esto se explica debido a que cuando la riqueza de especies de depredadores se incrementa, la representación de distintos tamaños del cuerpo de depredadores y comportamientos de caza se ampliarían, lo que a su vez aumentaría la probabilidad de que presas de diferentes tamaños y especies fueran capturadas por alguno de estos depredadores. Entre los invertebrados, muchos depredadores atacan a presas acordes con su tamaño relativo. Los depredadores además tienen comportamientos de captura asociados con un microhábitat en particular y que es visitado por algunas o una especie o incluso solo algún estadío larval de estas especies (Turnbull 1973, Post \& Riechert 1977). Los resultados de este 
trabajo coinciden con Provencher \& Riechert (1994) donde una mayor riqueza de especies incrementa el total de insectos consumidos. $\mathrm{Al}$ menos existen ocho factores que contribuyen potencialmente al efecto de esta asociación: el número de especies de depredador y presas; variaciones del tamaño del cuerpo entre las especies de depredadores y entre las especies de presas; variaciones del tamaño del cuerpo dentro de las especies de depredadores y dentro de las especies de presas; variaciones en el comportamiento de captura y variaciones en el ciclo de vida de la presa.

Las arañas cazadoras por emboscada son principalmente depredadoras generalistas de insectos y otras arañas y se encuentran típicamente en grandes asociaciones de especies (Post \& Riechert 1977, Riechert \& Lockley 1984). O. salticus fue la especie depredadora que resultó más eficiente en la captura de presas, especialmente larvas de lepidópteros, seguramente debido a su estrategia de caza, caracterizada por deambular activamente entre las hojas y tallos en la búsqueda de presas. El segundo lugar de consumo lo ocupó $M$. pallidus, que también es una araña cazadora pero a diferencia de $O$. salticus espera inmóvil al acecho a sus presas. Posiblemente, esta diferenciación de técnicas de captura explique los menores valores de consumo registrados. En el caso de Araneus sp. que depende de la movilidad de sus presas para que caigan en sus telas, fue la menos eficaz en la captura de larvas defoliadoras. En los tratamientos donde se incorporó a L. poliostoma se evidenció el consumo de otras presas como los coleópteros y ortópteros; esto debido a que los licósidos son arañas cazadoras corredoras de suelo, de mayor tamaño que las arañas cazadoras por emboscada, que habitan sobre la vegetación y son capaces de consumir presas de mayor tamaño (ortópteros) y de exoesqueletos más gruesos (curculiónidos y crisomélidos). Araneus sp. resultó ser menos eficaz en la captura de este tipo de insectos que, probablemente por ser una araña tejedora, esté más especializada en el consumo de presas voladoras tales como dípteros o himenópteros.
Del estudio realizado surge que las arañas presentan ciertas características que las constituyen como enemigos naturales capaces de ejercer o contribuir en el control de insectos plaga de los cultivos de alfalfa. Estas características se relacionan con el hecho de que las arañas son abundantes, aparecen durante todo el desarrollo fenológico de los cultivos, tienen posibilidades de sobrevivir largos períodos sin consumo de alimento y son capaces de alimentarse de algunas de las especies plaga que ocasionan serios daños a la vegetación.

\section{RESUMEN}

Las arañas son depredadoras capaces de reducir las poblaciones de insectos plaga en agroecosistemas. Para medir la selectividad frente a distintas presas, se realizaron ensayos de consumo diario, efecto de los depredadores aisladamente y en conjunto sobre el número de presas y efecto indirecto de los depredadores sobre la vegetación; se utilizaron jaulas experimentales de 1x1m cubiertas con una fina malla plástica. Misumenops pallidus, Oxyopes salticus y Araneus sp. fueron utilizadas como depredadores generalistas, áfidos gorgojos, larvas de lepidópteras, crisomélidos y ortópteros como sus presas potenciales. Las arañas prefirieron larvas de lepidópteros frente a curculiónidos y áfidos. El consumo máximo de larvas de lepidópteros fue de $93.33 \%$, áfidos $25.33 \%$ y curculiónidos $11.67 \%$. El Índice Q para las tres especies de arañas demostró una selectividad positiva sólo para las larvas defoliadoras. $O$. salticus presentó las tasas de consumo mayores. Rachiplusia nu fue la especie más consumida. El valor máximo de consumo registrado en 24 h fue para $O$. salticus frente a $R . n u(\mathrm{C})=2.8$. La asociación de varias especies de arañas depredadoras incrementó el total de insectos capturados. La presencia de arañas provocó la disminución del número de hojas dañadas por el efecto de las larvas de lepidópteros plaga.

Palabras clave: Araneae, plagas, agroecosistemas, enemigos naturales.

\section{REFERENCIAS}

Armendano, A. \& A. González. 2010. Comunidad de arañas (Arachnida, Araneae) del cultivo de alfalfa en Buenos Aires, Argentina. Rev. Biol. Trop. 58: 747-757.

Belmonte, M.L., N. Carrasco \& A. Báez. 2006. Cosecha Gruesa. Soja, Maíz, Girasol. Manual de campo 
Proyecto RIAP. EEA INTA Anguil "Ing. Agr. G. Covas" La Pampa Argentina.

Beltramo, J., I. Bertolaccini \& A. González. 2006. Spiders of soybean crops in Santa Fe Province, Argentina: Influence of surrounding spontaneus vegetation on lot colonization. Braz. J. Biol. 66: 29-41.

Beckerman, A.P., M. Uriarte \& O.J. Schmitz. 1997. Experimental evidence for a behavior- mediated trophic cascade in a terrestrial food chain. Proc. Natl. Acad. Sci. 94: 10735-10738.

Cheli, G., A. Armendano \& A. González. 2006. Preferencia alimentaria de arañas Misumenops pallidus (Araneae: Thomisidae) sobre potenciales insectos presa de cultivos de alfalfa. Rev. Biol. Trop. 54: 505-513.

Edwards, C.A., K.D. Sunderland \& K.S. George. 1979. Studies on polyphagous predators of cereal aphids. J. Appl. Ecol. 16: 811-823.

Feber, R.E., J. Bell, P.J. Johnson, L.G. Firbank \& D.W. MacDonald. 1998. The effects of organic farming on surface- active spider (Araneae) assemblages in wheat in Southern England, UK. J. Arachnol. 26: 190-202.

Foelix, R.F. 1982. Biology of spiders. Harvard University, Cambridge, EEUU.

Godfrey, K.E., W.H. Whitcomb \& J.L. Stimac.1989. Arthropod predators of velvetbean caterpillar, Anticarsia gemmatalis Hübner (Lepidoptera: Noctuidae) eggs and larvae. Environ. Entomol. 18: 118-123.

Gravena, S. \& W.L. Sterling. 1983. Natural predation on the cotton leafworm (Lepidoptera: Noctuidae). J. Econ. Entomol. 76: 779-784.

Greenstone, M.H. \& A.F. Bennett. 1980. Foraging strategy and metabolic rate in spiders. Ecology 61: 1255-1259.

Gregory, B.M., C.S. Barfield \& G.B. Edward.1989. Spider predation on velvetbean caterpillar moth (Lepidoptera: Noctuidae) in a soybean field. J. Arachnol. 17: 119-122.

Hairston, N.G, F.E. Smith \& L.B. Slobodkin. 1960. Community structure, population control, and competition. Am. Nat. 94: 421-425.

Harcourt, D.C., J.R. Aragón \& R. González. 1986. Plagas de la alfalfa, p. 98-104. In C. Bariggi, B.L. Marble, C.D. Itria \& J.M. Brun. Investigación, tecnología y producción de alfalfa. Colección Científica del INTA, Buenos Aires, Argentina.
Harwood, J.D., K.D. Sunderland \& W.O.C. Symondson. 2004. Prey selection by Linyphiid spiders: molecular tracking of the effects of alternative prey on rates of aphid consumption in the field. Molec. Ecol. 13: 3549-3560.

Jacobs, J. 1974. Quantitative measurement of food selection. A modification of the forage ratio and Ivlev's selectivity index. Oecologia 14: 413- 417.

Lang, A., J. Filser \& J.R. Henschel. 1999. Predation by ground beetles and wolf spiders on herbivorous insects in a maize crop. Agric. Ecosyst. Environ. 72: 189-199.

Lang, A. 2003. Intraguild interference and biocontrol effects of generalist predators in a winter wheat field. Oecologia 134: 144-153.

Lanteri, A.A. 1994. Bases para el control integrado de los gorgojos de la alfalfa. De la Campana, Buenos Aires, Argentina.

Lanteri, A.A., M. Loiacono, N. Díaz \& A. Marvaldi. 1998. Natural enemies of weevils (Coleoptera: Curculionoidea) harmful to crops in Argentina. Revta. Bras. Ent. 41: 517-522.

LeSar, C.D. \& J.D. Unzicker. 1978. Life history, habits, and prey preferences of Tetragnatha laboriosa (Araneae: Tetragnathidae): Environ. Entomol. 7: 879-884.

Liljesthröm, G., E. Minervino, D. Castro \& A. González. 2002. La comunidad de arañas del cultivo de soja en la provincia de Buenos Aires, Argentina. Neotrop. Entomol. 31: 197-209.

Losey, J.E. 1996. Synergism between ground and foliarforaging predators of aphids in alfalfa. Dissertation. University of Maryland, College Park, Maryland, EEUU.

Losey, J.E. \& R.F. Denno.1997. The escape response of pea aphids to foliar- foraging predators: factors affecting dropping behavior. Ecol. Entomol. 23: 53-61.

Marc, P.A., A. Canard \& F. Ysnel. 1999. Spiders (Araneae) useful for pest limitation and bioindication. Agric. Ecosyst. Environ. 74: 229-273.

Minervino, E. 1996. Estudio biológico y ecobiológico de arañas depredadoras de plagas de soja. Tesis Doctorado. Universidad de La Plata, La Plata, Argentina.

Nentwig, W. 1987. The prey of spiders, p. 249-263. In W. Nentwig. Ecophysiology of spiders. Berlin-Heidelberg, Alemania.

Nentwig, W. 1988. Augmentation of beneficial arthropods by strip-management: succession of predacious 
arthropods and long- term change in the ratio of phytophagous and predacious arthropods in a meadow. Oecologia 76: 597-606.

Nuessly, G.S. \& W.L. Sterling. 1994. Mortality of Helicoverpa zea (Lepidoptera: Noctuidae) eggs in cotton as a function of oviposition sites, predator species, and desiccation. Environ. Entomol. 23: 1189-1202.

Nyffeler, M., D.A. Dean \& L. Sterling. 1987. Evaluation of the importance of the striped lynx spider, Oxyopes salticus (Araneae: Oxyopidae), as a predator in Texas cotton. Environ. Entomol. 16: 1114-1123.

Nyffeler, M., W.L. Sterling \& D.A. Dean. 1994a. How spiders make a living. Environ. Entomol. 23: 1357-1367.

Nyffeler, M., W.L. Sterling \& D.A. Dean. 1994b. Insectivorous activities of spiders in United States field crops. J. Appl. Ent. 118: 113- 128.

Nyffeler, M. 1999. Prey selection of spiders in the field. J. Arachnol. 27: 317-325.

Nyffeler, M. \& K. Sunderland. 2003. Composition, abundance and pest control potential of spider communities in agroecosystems: a comparison of European and US studies. Agric. Ecosyst. Environ. 95: 579-612.

Parra, J.R.P. 1986. Cría de insectos para estudios con patógenos, p. 348-373. In S. Batista Alves. Control microbiano de insectos. San Pablo, Brasil.

Pedigo, L.P. 2001. Entomology and pest management. Prentice Hall, Nueva Jersey, EEUU.

Polis, G.A. \& D.R. Strong. 1996. Food web complexity and community dynamics. American Naturalist 147: 813-846.

Post, III, W.M. \& S.E. Riechert. 1977. Initial investigation into the structure of spider communities. I. Competitive effects. J. Anim. Ecol. 46: 729-749.

Provencher, L. \& S.E. Riechert. 1994. Model and field test of prey control effects by spider assemblages. Environ. Entomol. 23: 1-17.

Reed, T., M. Shepard \& S.G. Turnipseed.1984. Assessment of the impact of arthropod predators on noctuid larvae in cages in soybean fields. Environ. Entomol. 13: $954-961$

Richards, O.W. \& R.G. Davies. 1984. Tratado de entomología Imms. Clasificación y biología. Omega, Barcelona, España.
Riechert, S.E. \& R. Gillespie. 1983. Habitat choice and utilization in the web spinners, p. 1-12. In W.A. Shear. Web Building spiders. Stanford University, Stanford, EEUU.

Riechert, S.E. \& T. Lockley. 1984. Spiders as biological control agents. Ann. Rev. Entomol. 29: 299-320.

Riechert, S.E. \& J.M. Harp. 1987. Nutritional ecology of spiders, p. 645-672. In F. Slansky \& J.G. Rodriguez. Nutritional ecology of insects, mites and spiders. Nueva York, EEUU.

Riechert, S.E. \& L. Bishop. 1990. Prey control by an assemblage of generalist predators: spiders in garden test system. Ecology 71: 1441- 1450.

Riechert, S.E. \& K. Lawrence. 1997. Test for predation effects of single versus multiple species of generalist predators: Spiders and their insect prey. Entomol. Exp. Appl. 4: 147-155.

Ruberson, J.R. \& M.H. Greenstone. 1998. Predators of budworm/ bollworm eggs in cotton: an immunological study. Proc. Beltwide Cotton Conf. 2: 1095-1098.

Schmitz, O.J., A.P. Beckerman \& K.M. O'Brien. 1997. Behaviorally mediated trophic cascades: effects of predation risk on food web interactions. Ecology 78: 1388-1399.

Schmitz, O.J. \& K.B. Suttle. 2001. Effects of top predator species on direct and indirect interactions in a food web. Ecology 82: 2072-2081.

Schmitz, O.J. 2003. Top predator control of plant biodiversity and productivity in an old- field ecosystem. Ecology Letters 6: 156-163.

Schmitz, O.J. 2004. From mesocosms to the field: the role and value of cage experiments in understanding top- down effect in ecosystems, p. 277-302. In W.W. Weisser \& E. Siemann. Insects and ecosystem function. Berlin, Alemania.

Shaw, P.B., J.C. Owens, E.W. Huddleston \& D.B. Richman.1987. Role of arthropod predators in mortality of early instars of the range caterpillar, Hemileuca oliviae (Lepidoptera: Saturniidae). Environ. Entomol. 16: 814-820.

Sunderland, K.D. 1999. Mechanisms underlying the effects of spiders on pest populations. J. Arachnol. 27: $308-316$

Toft, S. 1995. Value of the aphid Rhopalosipum padi as food for cereal spiders. J. Appl. Ecol. 32: 552-560. 
Toft, S. 1999. Prey choice and spider fitness. J. Arachnol. 27: 301-307.

Trumper, E.V. 2001. Toma de decisiones en manejo de plagas en siembra directa, p. 205-212. In Panigatti et al. Siembra Directa, INTA, Buenos Aires, Argentina.

Turnbull, A.L. 1973. Ecology of true spiders (Araneomorphae). Annu. Rev. Entomol. 18: 305-348.

Vichitbandha, P. \& D.H. Wise. 2002. A field experiment on the effectiveness of spiders and carabid beetles as biocontrol agents in soybean. Agric. Forest Entomol. 4: 31-38.

Whitcomb, W.H. 1974. Predaceous insects, spiders and mites of Arkansas cotton fields. Ark. Agric. Exp. Stn. Bull. 690: 1-84.
Wise, D.H. 2006. Cannibalism, food limitation, intraspecific competition and the regulation of spider populations. Ann. Rev. Entomol. 51: 441-465.

Young, O.P. \& T.C. Lockley. 1986. Predation of striped lynx spider, Oxyopes salticus (Araneae: Oxyopidae), on tarnished plant bug; Lygus lineolaris (Heteroptera: Miridae): a laboratory evaluation. Ann. Entomol. Soc. Am. 79: 879-883.

Young, O.P. 1989. Field observations of predation by Phiddipus audax (Araneae: Salticidae) on arthropods associated with cotton. J. Entomol. Sci. 24: 266-273.

Young, O.P. \& G.B. Edwards. 1990. Spiders in United States field crops and their potential effect on crop pests. J. Arachnol. 18: 1-27. 